\title{
Preiskommunikation in Krisenunternehmen - eine Betrachtung aus Praxis-Sicht
}

\author{
Michael Paul
}

\begin{abstract}
Zusammenfassung
Unternehmenskrisen werden meist von Preiskrisen begleitet. Der Preis als „Fieberthermometer" offenbart Probleme, wie etwa mangelnde Wettbewerbsstärke oder nicht marktgerechte Angebote, häufig schon bevor die Krise insgesamt sichtbar wird. In der Krise selbst führt ein erheblicher Liquiditätsdruck nicht selten zu weiteren Preissenkungen. Dass sich die „Macht“ der Kunden und des Wettbewerbs gegenüber einem geschwächten Gegner vergrößert, verschärft die Herausforderung für das Management. Dieser Beitrag stellt ein systematisches Vorgehen zur Preiskommunikation in Krisenunternehmen vor, das mit der Definition der Preisziele und preispolitischen Maßnahmen beginnt und daraus den Stil und die Kanäle der Preiskommunikation ableitet. Dabei wird insbesondere auf die liquiditätsgetriebene Kurz- und die am Unternehmenswert und der nachhaltigen Rentabilität orientierte Mittelfristperspektive Bezug genommen.
\end{abstract}

\section{$1 \quad$ Einführung}

Der in Deutschland zur Analyse von Krisen allgemein angewandte Standard „Anforderungen an Sanierungskonzepte“ (IDW S 6) macht deutlich, dass es „die“ Krise nicht gibt. Vielmehr sind es verschiedene Krisenstadien (vgl. Abb. 1), die durch unterschiedliche Konstellationen bzw. Phasen gekennzeichnet sind und nicht einmal alle nach außen bekannt werden müssen (siehe IDW 2018, S. 6, Tz 31):

M. Paul ( $\square)$

paul und collegen consulting gmbh, Wien, Österreich

E-Mail: M.Paul@paulcollegen.com 


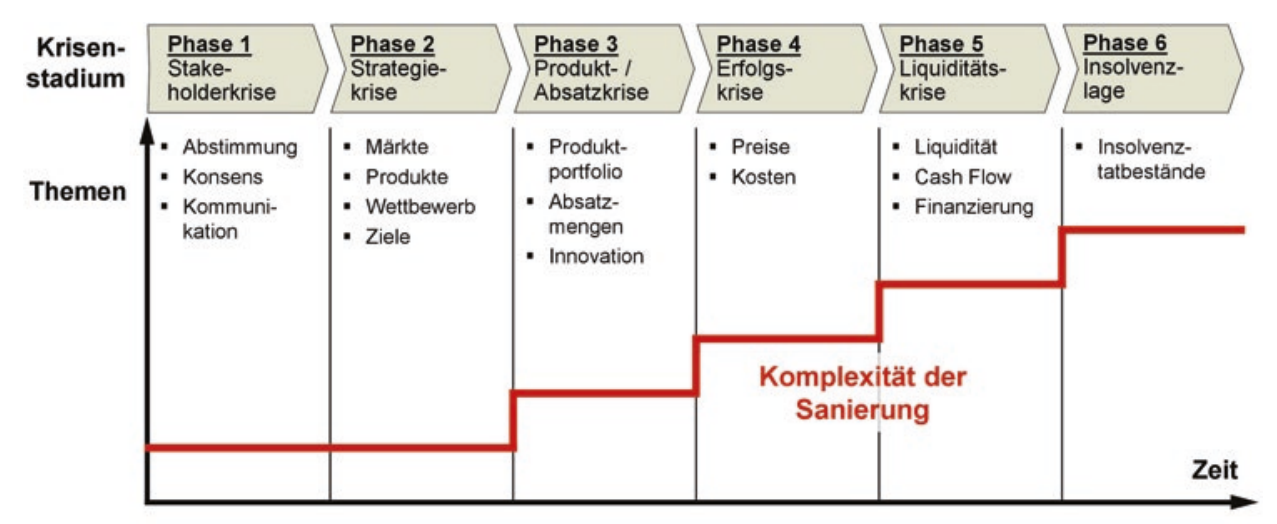

Abb. 1 Die 6 Phasen der Krisenstadien nach Häger und Hiltner (2017)

- Die Stakeholderkrise, die durch Uneinigkeit bzw. Handlungsunfähigkeit der Eigentümer gekennzeichnet ist.

- Die Strategiekrise, die davon geprägt ist, dass das Unternehmen über kein klares Geschäftsmodell mehr verfügt bzw. dieses nicht mehr funktioniert (z. B. aufgrund heftiger Disruptionen im Markt).

- Die Produkt- und Absatzkrise, in deren Verlauf das Unternehmen gegenüber dem Wettbewerb im Markt an Boden verliert und zumindest stagnierende Marktanteile realisieren muss.

- Die Erfolgskrise, in der es dem Unternehmen nicht mehr gelingt, im Wettbewerbsumfeld übliche Renditen zu erzielen bzw. sogar Verluste anfallen.

- Die Liquiditätskrise, in der die Zahlungsfähigkeit des Unternehmens nicht mehr gesichert ist.

- Die Insolvenzlage, in der das Unternehmen zahlungsunfähig ist und ein gesetzlich normiertes Verfahren der Sanierung durchlaufen muss.

Parallel zu den bekannten Krisenphasen verläuft in der weit überwiegenden Zahl von Unternehmen, die später möglicherweise in existenzbedrohende Situationen kommen, in den meisten Fällen eine sich verschärfende „Preiskrise“. Aus Sicht der Preispolitik nämlich ergeben sich häufig schon in den frühen Krisenphasen Probleme: Geringere Preisspielräume werden nicht selten schon in der Strategiekrise sichtbar. Eine unklare Positionierung, geringe Fokussierung im Sortiment oder den bearbeiteten Märkten etc. führen zum Abschmelzen eines Preispremiums oder verhindern, dass ein solches überhaupt erreicht werden kann. In den Phasen 3 bis 4 verschärft sich diese Problematik. Häufig werden in einer gewissen Panik unkontrolliert Preise gesenkt, um damit strategische Probleme sowie einhergehende Misserfolge zu überdecken. In diesem Beitrag stehen insbesondere die beiden letzten Phasen - 5 und 6 - im Mittelpunkt.

Die Situation des Unternehmens ist in diesen beiden Phasen anders als in den ersten in der Regel öffentlich bekannt. Das Management steht unter einem besonderen Druck: „Mit zu- 
nehmender Insolvenznähe steigt die Notwendigkeit, schnell greifende Sofortmaßnahmen umzusetzen. Wird eine akute Illiquidität festgestellt, müssen unverzüglich, d. h. innerhalb von längstens drei Wochen, Maßnahmen zu deren Beseitigung konkretisiert und umgesetzt werden“ (IDW 2018, S. 6 Tz 13). In dieser Situation des Handlungsdrucks müssen zahlreiche Aktionen gesetzt werden, von der Sicherung der Lieferantenbasis bis hin zu einer engen Überwachung der Liquidität. Dies erfordert eine klare Prioritätensetzung und Fokussierung auf überlebensnotwendige Aktionen von den Beteiligten. Tendenziell wird dabei eher auf die Ausgaben- als auf die Einnahmenseite geachtet, erwartet man sich doch hier schnellere Effekte. Das ist insbesondere deshalb problematisch, als die Umsatzseite und hier nicht zuletzt das Thema „Preis“ für eine Bewältigung der Krise von enormer Bedeutung sind, sowohl für die Überwindung der akuten Situation als auch für den Weiterbestand des Unternehmens. Dabei sieht sich das Management besonderen Rahmenbedingungen gegenüber.

\section{Mögliche Ziele der Sanierung als wesentliche Rahmenbedingung}

\subsection{Ziel der schnellen Wiedererlangung der Zahlungsfähigkeit}

Wesentliche Rahmenbedingung sind die Ziele, die von Eigentümern/Gläubigern, dem Insolvenzverwalter oder dem Management teilweise sogar parallel verfolgt werden (müssen).

Geht es darum, den Erhalt des Unternehmens in bisheriger Form durch schnelle Wiedererlangung der Zahlungsfähigkeit zu erhalten? „Sanierungsfähig ist ein erwerbswirtschaftliches Unternehmen nur dann, wenn [...] durch geeignete Maßnahmen auch nachhaltig die Wettbewerbsfähigkeit wiedererlangt werden kann (nachhaltige Fortführungsfähigkeit i. S. einer Sanierungsfähigkeit)“ (IDW 2018, S. 6, Tz 18). Nach einer Sanierung muss also auf jeden Fall wieder wirtschaftliches Arbeiten möglich sein. Dies erfordert auch die Erzielung von Preisen, die eine nachhaltige Fortführung ermöglichen. Nur über Kostensenkungen und Mengensteigerungen werden dauerhaft keine ausreichenden Cash-Flows oder gar eine im Marktvergleich übliche Rendite erzielbar sein. Schon im Zeitpunkt der Erstellung einer Fortbestehensprognose muss es deshalb einen klaren Plan geben, wie auch die Preispositionierung im Markt ,saniert“ werden kann. Opportunistische Preisvariationen wirken dem eher entgegen, selbst wenn sie kurzfristig helfen würden, die Liquidität zu verbessern. Das Ziel einer Fortführung erfordert also einen besonders sensiblen Umgang mit dem Thema „Preis“. Im Falle eines Herstellers von Haushaltsgeräten mit starker Marke war der hohe Lagerbestand mit entsprechender Kapitalbindung wesentlicher Teil der betriebswirtschaftlichen Krisenursachen. Im Sanierungsteam wurde deshalb intensiv darüber diskutiert, mittels eines Abverkaufs mit massiven Preissenkungen überhöhte Lagerbestände abzubauen und notwendige Liquidität zu generieren (vgl. Klein 2009, S. 69 sowie Helbing Gruppe 2009, S. 12-14). Starke Bedenken gingen in die Richtung, dass durch diese Aktion die Preispositionierung massiv geschwächt werden könnte. Bislang war es immer gelungen, sich aus den Preiskämpfen der Branche heraus zu halten. Es bestand die Befürchtung, dass nun durch diese „Sanierung“ eine neue Krisenursache geschaffen werden könnte. 


\subsection{Ziel des Verkaufs des Krisenunternehmens}

Ist ein Verkauf zumindest von Unternehmensteilen geplant? Hier gilt gleiches: Potenzielle Käufer des Unternehmens bzw. des Betriebs werden den Sinn der Übernahme selbst und den Wert des Unternehmens daran messen, welche zukünftigen Cash-Flows darin erzielbar sind (vgl. Spremann 2005, S. 6-7; Longstaff 2008, S. 2; Schüler 2012, S. 14). Preisaggressive Aktionen während der Phase der Krise wirken hier klar wertsenkend. Nicht nur, weil auf absehbare Zeit Umsätze unter einer beschädigten Preispositionierung und darüber hinaus gegebenenfalls noch der Sättigung des Marktes durch unkontrollierte Abverkäufe leiden. Wieder zu einer besseren Preispositionierung zu kommen, wird darüber hinaus unter diesen Rahmenbedingungen zusätzlichen Aufwand vor allem in der Kommunikation und im Vertrieb erzeugen (vgl. Homburg 2017, S. 87-88 und S. 738; Helbing Gruppe 2009, S. 12-14).

\subsection{Ziel der Liquidation des Unternehmens}

Steht eine Liquidation des Krisenunternehmens im Raum und geht es nur noch darum, möglichst hohe Quoten für die Gläubiger zu erzielen, sieht die Betrachtung anders aus als bei den Zielen 1 und 2. Im Spannungsfeld von Kundenpsychologie und der Notwendigkeit, Liquidität für die Gläubiger zu generieren, wird dann abzuwägen sein, wie das folgende Beispiel zeigt:

Als die letzten Kaufhäuser einer insolventen Gruppe geschlossen wurden, galt es, den Warenbestand von mehr als 50 Warenhäusern komplett abzuverkaufen. Für den Insolvenzverwalter war dies eine klare Abwägung: Von der Höhe des Abverkauferlöses sollte nicht unwesentlich abhängen, inwiefern Gläubiger noch bedient werden könnten und für die Belegschaft ein Sozialplan möglich sein würde. Sehr moderate Preissenkungen und damit höhere Preise, bzw. ein flacherer Verlauf der Preissenkungen, könnten möglicherweise zu einem besseren Liquidationsergebnis führen. Ebenso könnte es aber auch zu einer zu langen Phase des (Liquidität vernichtenden) Schließungsprozesses mit hohen Restbeständen wegen mangelnder Attraktivität für die Kunden kommen. Darüber hinaus stellte sich die Frage, wie lange man es schafft, Mitarbeiter und Leiter der Kaufhäuser im Wissen um das Ende des eigenen Arbeitsplatzes so zu motivieren, genau diesen Arbeitsplatz selbst aufzulösen? Radikale Preissenkungen hätten möglicherweise eine schnelle Schließung der Outlets ermöglicht, hätten aber zu geringen Erlösen geführt.

\section{$3 \quad$ Handlungsspielräume als weitere Rahmenbedingung}

Die zweite wesentliche Rahmenbedingung von Preiskommunikation folgt aus einer Verschiebung der Handlungsspielräume der Beteiligten in einer Insolvenzsituation (vgl. ThieBen 2013, S. 54). Sind die ersten Phasen einer Krise meist noch mit den üblichen Instrumenten der Preispolitik und Preiskommunikation zu bewältigen, kommt es in der Liquiditätskrise und erst recht in der Phase der Insolvenz zu einer Veränderung der (empfundenen und tatsächlichen) Stärke, die in der Preiskommunikation zum Handeln zwingt: 
- Verunsicherung und Erwartungen von Kunden. Kunden meinen, sie gingen gerade bei Käufen mit einer langfristigen Wirkung (z. B. langlebige Produkte mit einem Bedarf an Ersatzteilen, Service etc.) Risiken ein, wenn sie zu Angeboten eines sichtbar instabilen Anbieters greifen. Gleichzeitig nehmen Kunden ihre eigene Position in Krisenzeiten in der Regel als stärker wahr als in normalen Zeiten - das angeschlagene Unternehmen ist möglicherweise mehr als in der Vergangenheit auf die Umsätze angewiesen (vgl. ThieBen 2013, S. 57). Beides führt in der Regel zu der Erwartung höherer Preiszugeständnisse. In dem erwähnten Kaufhausbeispiel begannen die Kunden schon seit der Bekanntgabe von Problemen des Unternehmens sich danach zu erkundigen, wann denn jetzt endlich die Sonderangebote beginnen würden. Man müsse doch jetzt - so deren Erwartung - angesichts der schlechten Lage die Preise senken. Eine Erwartung, die sogar rationales Handeln ausschaltete: In den Tagen der Vorbereitung des Räumungsverkaufs, als zwar noch überall die regulären Preise galten, zunächst einmal aber die Schaufenster mit Plakaten behängt wurden, auf denen sich große Prozentzeichen befanden, stieg die Kundenfrequenz enorm an. In der Erwartung, das müsse doch alles schon vergleichsweise günstig sein, kauften viele Kunden in ungewöhnlichem Umfang ein, das Unternehmen realisierte Tagesumsätze wie seit Jahren nicht mehr.

- Wettbewerber sehen in den Problemen eines Konkurrenten eigene Chancen. Je nachdem, welche Effekte sie sich von einer Marktbereinigung versprechen, werden sie bereit sein, in dieser Marktphase in die Gewinnung von neuen Kundenbeziehungen zu investieren (zu diesem Denken der Geschäftsbeziehung, vgl. Plinke 1991). In der Regel werden sie dabei von der Hoffnung auf mittelfristig steigende Preise geleitet, nicht selten werden sie aber selbst preispolitisch aggressiv, um in einem durch das ,untergehende“ Unternehmen angezettelten Preiskrieg nicht zu den Verlierern zu gehören (Fürst 2004, S. 74; Rao et al. 2000). In der Insolvenz eines Wartungsdienstleisters begannen einzelne Wettbewerber schon in den Tagen nach Veröffentlichung des Insolvenzantrags damit, ihnen bekannte Kunden des Krisenunternehmens telefonisch anzusprechen und gezielt mit niedrigen Preisen und dem Hinweis auf vermeintliche Risiken beim bisherigen Dienstleister einen Wechsel herbeizuführen. Im Bewusstsein, dass die begrenzte Zahl von Kunden in diesem Segment jetzt einmal neu aufgeteilt wird, kamen sie einer Kommunikation des Krisenunternehmens zuvor. Einige aus dieser Gruppe überlegten sogar einen Einstieg im Krisenunternehmen und verfolgten dabei die Strategie eines niedrigen Kaufpreises durch gezielte Abschmelzung der Kundenbasis (der von den zukünftigen wirtschaftlichen Möglichkeiten des Unternehmens abhängt). Gänzlich unterschiedlich war die Vorgehensweise einiger anderer Wettbewerber: Diese planten zwar auch eine Übernahme des notleidenden Unternehmens, verhielten sich jedoch passiv. Das Image und die Wettbewerbsposition des Unternehmens, die bei einer zu starken „Beschädigung“ durch die Krise gefährdet gewesen wäre, wollten sie eher geschützt wissen.

- Das Management des Krisenunternehmens schließlich hat unter dem in dieser Situation dominanten Gesichtspunkt der Liquidität für einen ausreichenden Cash-Flow zu sorgen. Dies geschieht nicht selten aus einer Position der Schwäche heraus. Einzelne Lieferanten liefern, wenn überhaupt, nur noch gegen Vorkasse. Dadurch leidet die Lieferfähigkeit, 
Kundenzusagen können unter Umständen nicht mehr eingehalten werden, es droht die Lieferunfähigkeit. Dazu kommen die besagten Unsicherheitsprobleme bei Kunden. Naheliegend ist da der Versuch, mit Preissenkungen und der Botschaft der Preisgünstigkeit in der Kommunikation kurzfristig Umsätze zu steigern oder zumindest zu stabilisieren (vgl. Thießen 2013, S. 61-62). Dem sind allerdings Grenzen dadurch gesetzt, dass Unternehmen in Krisen nicht selten mit einer nicht wettbewerbsfähigen Aufwandseite zu kämpfen haben und mangels eines funktionierenden Rechnungswesens (ein Kennzeichen zahlreicher Krisenunternehmen) diesen Aufwand nicht einmal ausreichend genau beziffern können. Zu starke Preissenkungen können somit trotz höherer Umsätze zu negativen Cash-Flow-Effekten führen.

Zweite wesentliche Rahmenbedingung preispolitischer Aktionen in der Krise ist also die Asymmetrie der Handlungsspielräume und Erwartungen der Akteure.

\section{$4 \quad$ Elemente der Preispolitik in der Krise - Grundlage und Inhalt der Preiskommunikation}

Die Bewältigung der Preiskrise erfordert eine eigene, klare Strategie, die im Einklang mit den Zielen und Rahmenbedingungen sowie vor allem dem gewählten Lösungsansatz zur Bewältigung der Unternehmenskrise stehen muss. Sie ist die Grundlage des Vorgehens in der Preiskommunikation.

In Anlehnung an Diller (Diller 2003, S. 210) lassen sich die preispolitischen Optionen allgemein folgendermaßen darstellen (darunter immer die jeweiligen Besonderheiten in der Krise, vgl. Abb. 2).

\begin{tabular}{|c|c|c|c|}
\hline & $\begin{array}{l}\text { Ausgestaltung des } \\
\text { Preissystems }\end{array}$ & $\begin{array}{l}\text { Preispositionierung } \\
\text { und -Segmentierung }\end{array}$ & $\begin{array}{l}\text { Strategische } \\
\text { Preiskoordination }\end{array}$ \\
\hline 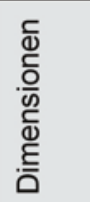 & $\begin{array}{l}\text { - Preisformen } \\
\text { - Preisfindung } \\
\text { - Preis- und } \\
\text { Konditionenkomponenten } \\
\text { - Preisdifferenzierung }\end{array}$ & $\begin{array}{l}\text { - Generische } \\
\text { Preispositionierung } \\
\text { - Kundennutzenkonzepte } \\
\text { - Preisstrategische } \\
\text { Wettbewerbskonzepte } \\
\text { - Zielgruppen }\end{array}$ & $\begin{array}{l}\text { - Preislinienpolitik } \\
\text { - Life-Cycle-Pricing } \\
\text { - Internationale } \\
\text { Preiskoordination }\end{array}$ \\
\hline 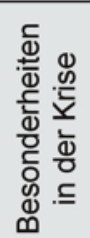 & $\begin{array}{l}\text { - Änderungen Kalkulation } \\
\text { - Preisfindung Änderung } \\
\text { - Aussetzen Preis- und } \\
\text { Konditionsbestandteile } \\
\text { - Anderes Preiskomponenten } \\
\text { - Stärkere Differenzierung }\end{array}$ & $\begin{array}{l}\text { - Änderung Positionierung } \\
\text { - Beziehung als } \\
\text { Nutzenkomponente } \\
\text { - Segment situativ neu } \\
\text { bewerten }\end{array}$ & $\begin{array}{l}\text { - Internationale } \\
\text { Differenzierung } \\
\text { - Aufgabe von Preislinien }\end{array}$ \\
\hline
\end{tabular}

Abb. 2 Preispolitische Optionen nach Diller (vgl. Diller 2003, S. 210) 
- Wichtigster Ansatzpunkt bei der Ausgestaltung des Preissystems ist die Änderung der Preiskalkulation bzw. der Preisfindung. Im Umfeld einer Unternehmenskrise wird sie sehr viel stärker situative Faktoren mit einbeziehen müssen. Die Notwendigkeit, Liquidität zu generieren, führt z. B. zu einer veränderten Sichtweise auf die Preisuntergrenze. Das folgende Beispiel skizziert diesen Zusammenhang: Ein traditionsreiches Unternehmen des Anlagenbaus befand sich in einer akuten Liquiditätskrise. Es stand die Vergabe des Baus einer Großanlage im asiatischen Raum an. Der Umsatz wäre der höchste der Unternehmensgeschichte gewesen. Die Laufzeit des Projektes sollte sich über 4 Jahre erstrecken. Es war klar: Wenn man diesen Auftrag zu einem einigermaßen akzeptablen Preis bekäme, wäre dies eine gute Grundlage für die Sicherung der Liquidität. Eine herkömmliche Vollkostenkalkulation erwies sich unter diesen Umständen für die Ermittlung der Preisuntergrenze als untauglich. Mit hoher Wahrscheinlichkeit wäre mit einem derart ermittelten Preis der Auftrag nicht zu erlangen gewesen. Man entschied sich deshalb dazu, die Preisuntergrenze liquiditätsorientiert zu ermitteln. Es wurden dabei die ,relevanten Kosten“ betrachtet, d. h. die mit der Herstellung verbundenen, zusätzlichen Kosten, die auch ausgabenwirksam sind. Die Möglichkeiten, kurzfristig die Personalkosten oder bestimmte Gemeinkostenbestandteile zu senken, erwiesen sich hingegen als beschränkt. Sie wurden deshalb, soweit nicht beeinflussbar, aus der Betrachtung herausgenommen. So wurde eine Preisuntergrenze ermittelt, unterhalb derer sich die Liquiditätssituation des Unternehmens verschlechtern und oberhalb derer eine Verbesserung eintreten würde. Eine solche Veränderung der Preisfindung führt nicht zu dauerhaft wirtschaftlichen Preisen, gibt aber die notwendigen Informationen, um situativ preispolitisch reagieren zu können.

- Ebenfalls situativ kann es Sinn machen, in der Krise Preis- und Konditionenbestandteile auszusetzen oder zu verändern. Gerade in Märkten mit wenigen Anbietern besteht auf Seiten der Kunden unter Umständen ein großes Interesse am Überleben des Krisenunternehmens. Nicht selten ist in solchen Fällen feststellbar, dass auf Seiten der Kunden sogar Bereitschaft zu einem ,Sanierungsbeitrag“ besteht. Dabei sind die Kunden bereit, entweder auf Konditionenbestandteile zu verzichten oder höhere Preise zu bezahlen. So ist es nicht unüblich, dass Zahlungsfristen verkürzt, Rabatte ausgesetzt oder Preise sogar erhöht werden können. Der Sanierungsbeitrag kann - zeitlich befristet sogar ein eigener Konditionenbestandteil werden.

- Nicht zuletzt kann die Veränderung der Kalkulation aber auch die Aussetzung bzw. Veränderung von Preiskomponenten zu einer stärkeren Differenzierung der Preise führen:

- Will man nicht flächendeckend mit niedrigeren Preisen in den Markt gehen, macht es unter Umständen Sinn, dies nach einzelnen Kundensegmenten wesentlich differenzierter zu tun. Dabei ist es sowohl möglich, dass gerade bislang wesentliche Kunden in den Genuss von preislichen Vorteilen kommen als auch die Erschließung völlig neuer Segmente mit günstigen Angeboten. Eine undifferenzierte Preissenkung findet häufig bei Abverkäufen im Einzelhandel statt. Dabei ist nicht selten zu beobachten, dass stärker bisherige Nicht-Kunden angesprochen werden, die preisorientierter kaufen, als bisherige Stammkunden. Im Falle einer Fortführung kann 
das erheblich negative Folgen haben. Ein vielfach erprobtes Vorgehen besteht deshalb darin, die preisaggressiven Segmente nicht durch Angebote in den eigenen Geschäften anzusprechen. Die Preisdifferenzierung wird in diesem Fall durch eine Segmentierung der Vertriebswege untermauert. Umgesetzt wird dies, indem Waren entweder in anderen Vertriebsschienen zu wesentlich niedrigeren Preisen angeboten werden oder sogar im Ausland ,abgeschleust“"werden.

- Eine weitere Form der Differenzierung ist mit dem angesprochenen Konzept des Sanierungsbeitrags verbunden. Dabei werden Kunden, die bereit sind einen Sanierungsbeitrag zu leisten, für die Zeit nach der erfolgreichen Sanierung Vergünstigungen eingeräumt. Der Sanierungsbeitrag ist in diesem Falle eine Art Darlehen, das den Kunden über niedrigere Preise in den folgenden Jahren zurückgezahlt wird. Eine solche Form der Preisdifferenzierung erfordert die Existenz von Kundensegmenten, die zum einen ein großes Interesse am Überleben des Unternehmens und zum anderen eine so langfristige Perspektive haben, dass sie bereit sind, sich auf ein solches Preismodell einzulassen.

Eine Sanierung erfordert immer auch eine Hinterfragung der Preispositionierung und -segmentierung. Das kann in zwei Richtungen gehen:

- Ein bewusstes Anheben des Preisniveaus, um sich von unrentablem Geschäft trennen zu können (vgl. Fürst 2004, S. 50 und 78). Dies setzt voraus, dass das Unternehmen ausreichend flexibel auf der Kostenseite ist, um kurzfristig auch geringere Mengen kostengünstig produzieren zu können (vgl. Meffert 2013, S. 144-145). Weiterhin muss eine ausreichend große Gruppe von Kunden existieren, die bereit ist, auch ein höheres Preisniveau zu akzeptieren. Eine solche Strategie verfolgte ein Hersteller von Spezialmaschinen. Seine Produktlinien umfassten am unteren Ende des (Preis-)Spektrums relativ einfache Maschinen, bei deren Angebot man einem breiten Wettbewerb ausgesetzt war. Sie waren in den letzten 20 Jahren im Zuge einer Expansionsstrategie eingeführt worden. Die Idee war damals, die Rentabilität des Unternehmens dadurch zu erhöhen, dass man das bestehende Know-how und unterausgelastete Produktionskapazitäten nutzen könnte, um in diesem Massenmarkt zusätzliche Deckungsbeiträge zu erzielen. Tatsächlich hatten die Angebote in diesem Segment eher den Effekt, dass man unbewusst eine Art Preiskrieg bei den Basismaschinen auslöste. Günstige Maschinen mit dem starken Markennamen stießen in diesem Segment auf so starke Nachfrage, dass Wettbewerber begannen, noch stärker die Preise zu senken. Damit wurden nicht nur keine zusätzlichen Deckungsbeiträge mehr erzielt, sondern aufgrund der relativ teuren Produktion Verluste realisiert. Mit einer generellen Anhebung des Preisniveaus wollte man sich wieder stärker auf jenes Kundensegment konzentrieren, das qualitäts- und markenbewusst beschafft. Außerdem sollte damit der Ausstieg aus dem Basissegment erfolgen. Möglich war dies allerdings nur, weil die Produktion kurzfristig so umstrukturiert werden konnte, dass die verbleibende Fertigung in einem Werk konzentrierbar und die Schließung aller übrigen Werke möglich war. 
- Ein Absenken der Preisniveaus, um kurzfristig neue Kundengruppen und damit höheren Umsatz erzielen zu können. Wie bereits mehrfach erwähnt, ist dies die am häufigsten gewählte Option im Falle einer Unternehmenskrise (vgl. Fürst 2004, S. 72). Sie erfordert neben einer hohen Transparenz über die kalkulatorischen Zusammenhänge und das Preisverhalten der Kunden (z. B. wie ist die Preiselastizität, das heißt, wie stark reagieren Kundengruppen tatsächlich auf niedrigere Preise? Wie entwickeln sich die Kosten im Vergleich zu den Preiseffekten bei steigenden Mengen?) insbesondere eine Einschätzung der mittelfristigen Effekte (vgl. Fürst 2004, S. 74; Klein 2009, S. 35, 57-61). Ein typisches Beispiel: Eine Modemarke, die im Rahmen einer Insolvenz ihre Preispositionierung deutlich veränderte, um insbesondere hohe Lagerbestände abzubauen, ging zum Beispiel schon wenige Jahre später wiederum in die Insolvenz. Es war nicht gelungen, nach der Sanierung wieder in die ehemalige Luxuspositionierung vorzudringen. Ein Ausweg aus diesem Dilemma scheint ebenfalls die Preisdifferenzierung zu sein. Wenn schon mit niedrigeren Preisen operiert werden soll, so sollte dies in Segmenten oder Märkten passieren, die nicht zum Kern des Geschäftsfeldes des Unternehmens gehören.

Bei den Kundennutzen-Konzepten macht es Sinn, so wie beim Abstellen auf einen „Sanierungsbeitrag“, in der Argumentation gegenüber den Kunden den Nutzen des Erhalts des Krisenunternehmens zu verdeutlichen. Inhaltlich wird man dem Kunden darstellen, dass die Existenz einer größeren Auswahl an Anbietern für ihn auch einen zumindest ansatzweise monetär bewertbaren Nutzen hat. Ein Anbieter aus dem Bereich der Sicherheitstechnik, der durch Missmanagement in die Zahlungsunfähigkeit geraten war, konnte zum Beispiel bei seinen Kunden erfolgreich darstellen, dass es ohne sein Unternehmen in zahlreichen Projekten in Deutschland nur noch drei Anbieter geben würde (,,wir sorgen für Wettbewerb und faire Preise!" ). Dieses Argument verfing bei einer Reihe von Kunden, die bereit waren, zumindest vorübergehend für sie ungünstigere Konditionen zu akzeptieren. Eine solche Argumentation birgt allerding die Gefahr, dass Kunden Risiken, die sich aus Problemen der Langfristigkeit oder Verlässlichkeit ergeben, stärker gewichten (vgl. Thießen 2013, S. 57-58). Dies haben zum Beispiel schon mehrfach Finanzdienstleister oder Reiseanbieter erfahren müssen. Mit dem Aufkommen erster Gerüchte über Schwierigkeiten entstand ein sich selbst verstärkender Prozess: die Kunden hatten Sorge, dass ihre Einlagen bzw. Anzahlungen für zukünftige Reisen nicht mehr sicher seien. Dies führte zu einem weiteren Rückgang des Geschäfts, der wiederum zu zusätzlichen negativen Meldungen führte. Auf den Erhalt der Beziehung als Nutzenargument zu setzen, bedingt also zumindest auch eine Strategie, wie man empfundene Risiken beim Kunden abbauen möchte.

Die Neubewertung von Zielsegmenten wurde bereits mehrfach angesprochen. Wesentlich dabei ist die Beurteilung der Reaktion der Segmente auf preispolitische Aktionen und eine Einschätzung der aktuellen und zukünftigen Bedeutung für das Unternehmen. Verkürzt gesprochen, rücken Segmente in den Mittelpunkt der Betrachtung, mit denen kurzfristig Liquidität erzielbar ist, ohne für die Zukunft preispolitische Spielräume zu sehr einzuengen. Dass dafür unter Umständen höhere „Zäune“ zwischen den Segmenten aufgebaut werden müssen, etwa durch Nutzung anderer Vertriebswege, wurde bereits dargestellt. 
Einer der „Zäune“ zwischen Marktsegmenten, die mit mehr oder weniger aggressiver Preispolitik angesprochen werden, könnte die räumliche Distanz sein. Ein international tätiger Porzellanwarenhersteller wollte sein hohes Preisniveau in Europa, Nordamerika und Asien nicht gefährden. Im arabischen Markt hingegen verfügte man aufgrund geringer Distribution über eine nur schwache Marktstellung. Eine wesentliche Idee der Sanierung bestand darin, gemeinsam mit einem arabischen Großhändler in dortigen Shoppingmalls die bekannte Marke zu einem deutlich günstigeren Preisniveau anzubieten.

Dies erfordert wiederum - letztes Element der Preisstrategie - eine bewusste, krisenbezogene strategische Preiskoordination.

Wesentlich war so auch im Fall des Porzellanwarenhersteller die Koordination. So galt es zum Beispiel zu vermeiden, dass Zwischenhändler im arabischen Raum größere Mengen aufkaufen, um diese doch wieder in den europäischen Markt zu bringen. Dies erfolgte insbesondere über eine bewusste Steuerung der Preisabstände zwischen den verschiedenen regionalen Märkten („Preiskorridore“).

\section{$5 \quad$ Vorgehensweisen der Preiskommunikation in der Krise}

Die Preiskommunikation in der Krise muss im Gesamtrahmen der Krisenkommunikation gesehen werden. „Unter Krisenkommunikation wird die gezielte Informationsweitergabe an Adressaten nach dem Eintritt einer Krise verstanden. Daher zählt sie zu den ausgewählten taktischen Maßnahmen [...]. Dabei möchte eine Unternehmensführung Details der Öffentlichkeit bewusst vermitteln oder vorenthalten." (Katzdobler 2014, S. 30).

Daraus ergibt sich die grundsätzliche Einteilung der Kommunikationsstile in der Krise in einen aktiven und passiven Stil:

- Der aktive Stil ist dadurch gekennzeichnet, dass das Unternehmen seine Lage und die daraus resultierenden Aktionen in der Öffentlichkeit offen thematisiert und dabei versucht, Fragen und Erwartungen des Marktes vorwegzunehmen.

- Der passive Stil hingegen reagiert nur auf Informationsanforderungen des Marktes.

Vielfach wird in der Literatur ein aktiver Kommunikationsstil als überlegen dargestellt. Dies wird im Wesentlichen damit begründet, dass die Entstehung von Gerüchten gar nicht zu vermeiden ist, und durch ein proaktives Vorgehen Inhalte und Erwartungen besser steuerbar sind (vgl. Evertz und Krystek 2014, S. 235; Katzdobler 2014, S. 87; Fürst 2004, S. 60). In Fällen, in denen die Kommunikation des Unternehmens immer nur nachvollzogen hat, was ohnehin schon in der Öffentlichkeit bekannt war, zeigte sich häufig, dass die Glaubwürdigkeit des Managements gelitten hat (vgl. Thießen 2013, S. 60-57). Selbst zutreffende Aussagen werden nicht mehr ohne weiteres akzeptiert, da die Erfahrung aller Stakeholder dahin geht, dass sich immer wieder im Nachhinein ein Abweichen der Wirklichkeit von der vorherigen Kommunikation zeigt. Da zu diesen Stakeholdern nicht nur die Kunden und Wettbewerber des Unternehmens gehören, sondern auch dessen 
Arbeitnehmerinnen und Arbeitnehmer, besteht das große Risiko einer passiven Kommunikation in einem Verlust der internen Steuerungsmöglichkeiten im Unternehmen (vgl. Behringer 2017, S. 118; Thießen 2013, S. 63-67).

Trotzdem kann in bestimmten Konstellationen auch ein passiver Kommunikationsstil der Krise angemessen sein. Insbesondere dann, wenn negative Meldungen noch beherrschbar erscheinen und das Unternehmen nach außen noch handlungsfähig auftreten kann. Geht es um besonders vertrauenssensible Leistungen (zuvor wurden ja bereits bespielhaft Finanzdienstleistungen, Reiseangebote oder Serviceunternehmen erwähnt), so entwickelt sich unter Umständen bei Bekanntwerden negativer Entwicklungen eine Eigendynamik, die nicht mehr beherrschbar sein könnte. In solchen Konstellationen kann auch ein eher passiver Kommunikationsstil angezeigt sein.

Grundsätzlich sind auch Kombinationen beider Kommunikationsstile eine mögliche Option in der Preiskommunikation.

Unabhängig davon, ob wegen der Krise Veränderungen in der Preispolitik vorgenommen werden oder nicht, ist dies eine Information mit Signalwirkung. Entweder wird kommuniziert, dass an der bisherigen Linie festgehalten wird, was z. B. als Signal der Stärke aufgenommen werden kann, oder dass sich das Preisniveau ändert, was als Signal zum Handeln (mehr kaufen, anders kaufen) in den Markt wirken kann. Dieses Signal kann in verschiedener Weise verbreitet, bzw. bewusst thematisiert werden (vgl. Simon und Fassnacht 2009, S. 71). Dabei bieten sich dem Unternehmen dieselben Optionen der Preiskommunikation wie bei einem Unternehmen im „normalen Modus“ an, wobei sich diese in zwei große Gruppen unterteilen lassen:

- Die Preiswerbung. Diese umfasst sämtliche Maßnahmen, in denen der Markt durch den Einsatz verschiedener Medien über den Preis informiert wird. Diller spricht hier auch von der „Preisdeklaration“, sie „,umfasst alle schriftlichen Ausweise des jeweils gültigen Preises am Produkt oder am Verkaufsregal (,Preisauszeichnung“) sowie in Preislisten, Prospekten, Katalogen und anderen Werbemitteln des Anbieters“ (vgl. Diller 2003, S. 405).

- Die persönliche Preisvereinbarung. Hiermit sind sämtliche Maßnahmen umfasst, in denen ein Vertrieb Kunden entweder ergänzend zur Preiswerbung oder stattdessen Informationen zum Preis mitteilt und diesen Preis mit dem Kunden verhandelt (vgl. ThieBen 2013, S. 63-66).

Kombiniert man den aktiven bzw. den passiven Kommunikationsstil und die beiden Optionen der Umsetzung der Preiskommunikation als Preiswerbung oder persönliche Preisvereinbarung, so ergibt sich das in Abb. 3 gezeigte Bild.

Eine passive Preiswerbung könnte in der praktischen Umsetzung etwa in einer Anpassung von Preislisten und Preisauszeichnungen bestehen, sollte dies auf Druck des Marktes notwendig sein. Von einer passiven „Werbung“ zu sprechen, mag paradox klingen. Doch selbst, wenn Anpassungen beispielsweise der Preislisten oder Preisauszeichnungen ,aktiv“ vorgenommen werden, so erfolgt dies doch in einem Rahmen, der nicht 


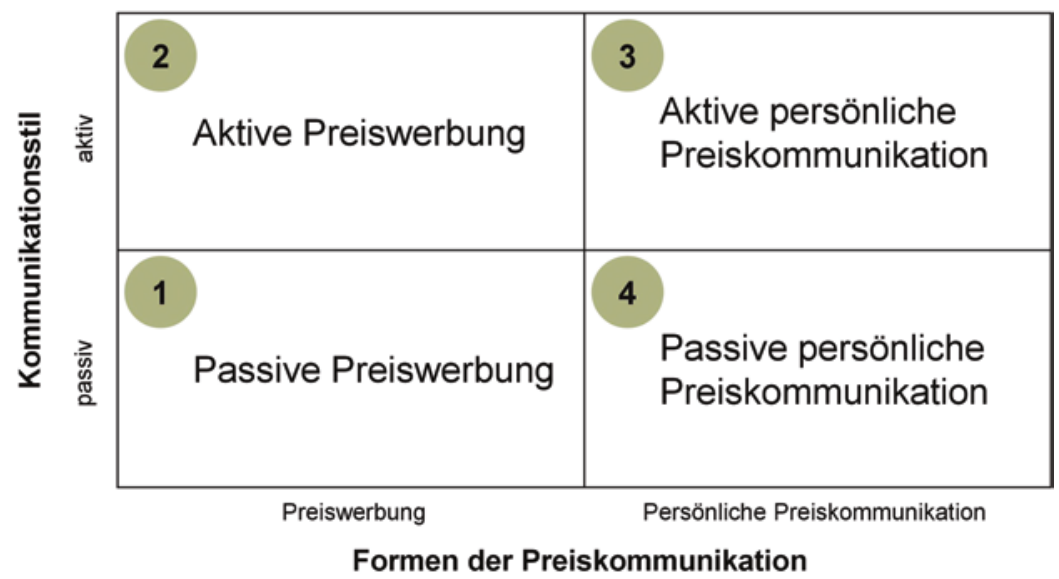

Abb. 3 Matrix Kommunikationsstil und Preiskommunikation

zwangsläufig zu einer größeren Aufmerksamkeit in einer breiteren Marktöffentlichkeit führt. In der praktischen Umsetzung würde das Unternehmen z. B. die Entwicklung des Umsatzes in der Krise weiter beobachten und nur dann Anpassungen vornehmen, wenn es hier zu erheblichen negativen Reaktionen kommt. Ein solches Vorgehen hat den Vorteil, dass zunächst einmal „Business as usual“ in den Markt ausgestrahlt und die Lage als „unter Kontrolle“ dargestellt wird. Dieser Vorteil ist zugleich auch der größte Nachteil eines solchen Vorgehens. Insbesondere wenn es darum geht, durch preisseitige Aktionen zusätzliche Liquidität durch höhere Umsätze zu schaffen, oder größere Warenbestände abzuverkaufen, ist dieses Vorgehen ungeeignet. Es eignet sich insbesondere dann, wenn man zumindest in einzelnen Marktsegmenten keinerlei Veränderung an der Preispolitik in der Krise vornehmen möchte. Selektiver lässt sich dieser Ansatz im E-Commerce verfolgen, je besser die Daten des Unternehmens über seine Kunden sind, desto selektiver können Preise angepasst werden. Je nach Informationsbedürfnissen und Preisbereitschaften kann dann sehr viel gezielter gesteuert werden.

Eine aktive Preiswerbung hingegen setzt unterschiedliche Medien ein, um Wahrnehmung für veränderte Preise zu schaffen. Im Gegensatz zur passiven Vorgehensweise wird sie eine veränderte Preisgünstigkeit besonders hervorheben. Bei allen Strategien, die darauf setzen, zusätzliche Umsätze zu generieren bzw. Warenbestände abzubauen, wird sie das Mittel der Wahl sein (vgl. Simon und Fassnacht 2009, S. 71). Sie muss allerdings nicht nur bei Preisänderungen zum Einsatz kommen. Auch die breite Kommunikation, dass ein Überleben des Unternehmens für den Kunden von Nutzen ist bzw. dass sich aus der aktuellen Situation keine Risiken ergeben, kann den Einsatz aktiver Preiswerbung erfordern, zum Beispiel auch im Sinne einer Solidarisierung zwischen Kunden und Unternehmen. Aufgrund ihrer Breitenwirkung darf die Wirkung aktiver Preiswerbung im Sinne eines „Signaling“ in Richtung des Wettbewerbs nicht unterschätzt werden Kap. , 
Signaling gegenüber Wettbewerbern - Erkennen und Verhindern von Preiswettbewerb und „Preiskrieg“““. Durch die breite Streuung und einfache Auffindbarkeit sendet die aktive Preiswerbung aufmerksamkeitsstarke Signale. Reaktionen bis hin zur angesprochenen Gefahr des Preiskrieges werden wahrscheinlicher.

Die persönliche Preiskommunikation, sowohl aktiv als auch passiv, funktioniert nur in solchen Branchen, in denen es direkte, persönliche (vertriebliche) Kontakte zu Kunden gibt. Sie spielt deshalb insbesondere bei hochwertigeren Konsumgütern, immer seltener im klassischen Einzelhandel, eher im personalisierten Online-Handel und in vielen Bereichen des B2B eine zentrale Rolle.

Die aktive Variante der persönlichen Preiskommunikation spielt vor allem dort eine Rolle, wo es um die Vereinbarung von Sanierungsbeiträgen oder der Modifikation von Konditionen geht. Wesentliche Voraussetzung solcher Vereinbarungen ist das Vertrauen der Kunden. Sie werden nur dann bereit sein, einem Unternehmen trotz Krise Zugeständnisse zu machen, wenn sie eine Gewähr dafür haben, dass sich diese auch auszahlen. Dies setzen die Verlässlichkeit des Managements und eine hohe Wahrscheinlichkeit des Überlebens voraus. Insofern ist das aktive Zugehen auf die Kundenunternehmen essenziell für den Erfolg dieses Vorgehens. Die Kunden müssen das Gefühl haben, dass sie vom Management des Krisenunternehmens immer rechtzeitig, möglichst mit als erste verlässliche Informationen erhalten. Dazu gehören eine transparente Darstellung der Unternehmenssituation und konkrete Vorschläge zum Beitrag der entsprechenden Kundenunternehmen. Deshalb setzt ein Einsatz aktiver persönlicher Preiskommunikation voraus, dass die im vorhergehenden Abschnitt dargestellten strategischen Optionen bereits durchdacht sind und dem Kunden konkrete Vorschläge unterbreitet werden können. Selbst wenn es nicht um Sanierungsbeiträge geht, sondern lediglich um ein Halten des Preises oder ein maßvolles Absinken, spielt die persönliche Kommunikation als wesentliche Maßnahme der Reduktion des empfundenen Risikos von Kunden eine entscheidende Rolle. Dies erfordert entweder vertriebliche Kontakte auf der Arbeits-, oder sogar Gespräche auf Managementebene. Insofern verschiebt sich an dieser Stelle nicht selten die Durchführung der Preiskommunikation in der Hierarchie. Sie wird zur „Chefsache“. Diese Einschaltung der Top-Ebene des Krisenunternehmens ist insbesondere wegen der Bedeutung des Vertrauens wichtig. Zugeständnisse oder Vereinbarungen mit einer gewissen Bindungswirkung werden nur akzeptiert werden, wenn die Kompetenz der Verhandelnden außer Frage steht. Befindet sich das Unternehmen bereits in der Insolvenz, wird sogar die Kompetenz des Managements in vielen Fällen nicht ausreichen. Es wird dann häufig von Kunden die Mitwirkung der Insolvenzverwaltung in der Verhandlung erwartet.

Die passive persönliche Preiskommunikation schließlich bietet sich dann an, wenn in den erwähnten Branchen und Märkten noch keine breiteren Reaktionen auf die Krise erkennbar sind. In diesen Fällen kann es möglicherweise Sinn machen, nur dann in persönlichen Gesprächen gezielt zu reagieren, wenn entsprechende Anfragen von Seiten der Kunden kommen. In der praktischen Umsetzung zeigt sich allerdings häufig, dass ein solches Vorgehen nur in Ausnahmefällen und dann auch nur für einen begrenzten Zeitraum 
sinnvoll ist. Je besser der Informationsstand der Kunden ist, desto mehr Anfragen werden das Unternehmen erreichen. Dies kann so massiv werden, dass es zu vertrieblichen Kapazitätsengpässen kommen kann.

\section{$6 \quad$ Ein systematischer Ansatz zur Ermittlung der Handlungsspielräume}

Ein systematisches Vorgehen preispolitischer Kommunikationsspielräume in der Krise muss zwischen einer kurzfristigen und einer mittelfristigen Perspektive unterscheiden (Abb. 4).

Die kurzfristige Perspektive wird in der Krise immer durch den Fokus auf die Liquidität geprägt sein. Dabei stellt sich die zentrale Frage, ob preispolitische Aktionen an der Liquidität überhaupt etwas ändern können.

Deshalb beginnt die Analyse mit den Kunden des Unternehmens:

- Wie ist die momentane Preispositionierung des Unternehmens? Wird sie als angemessen wahrgenommen oder ist sie bereits Teil der Krisenursachen? Herrscht möglicherweise schon längst ein Preiskrieg zwischen verschiedenen Anbietern, so dass die bestehende Preisbereitschaft der Kunden nicht mehr ausgeschöpft werden kann?

- Wie stark reagieren sie auf Preisänderungen? Würden Preisvariationen überhaupt zu Veränderungen des Umsatzes führen? Je starrer sich die Nachfrageseite verhält, desto geringere Chancen bestehen, hier kurzfristige Änderung herbeizuführen.

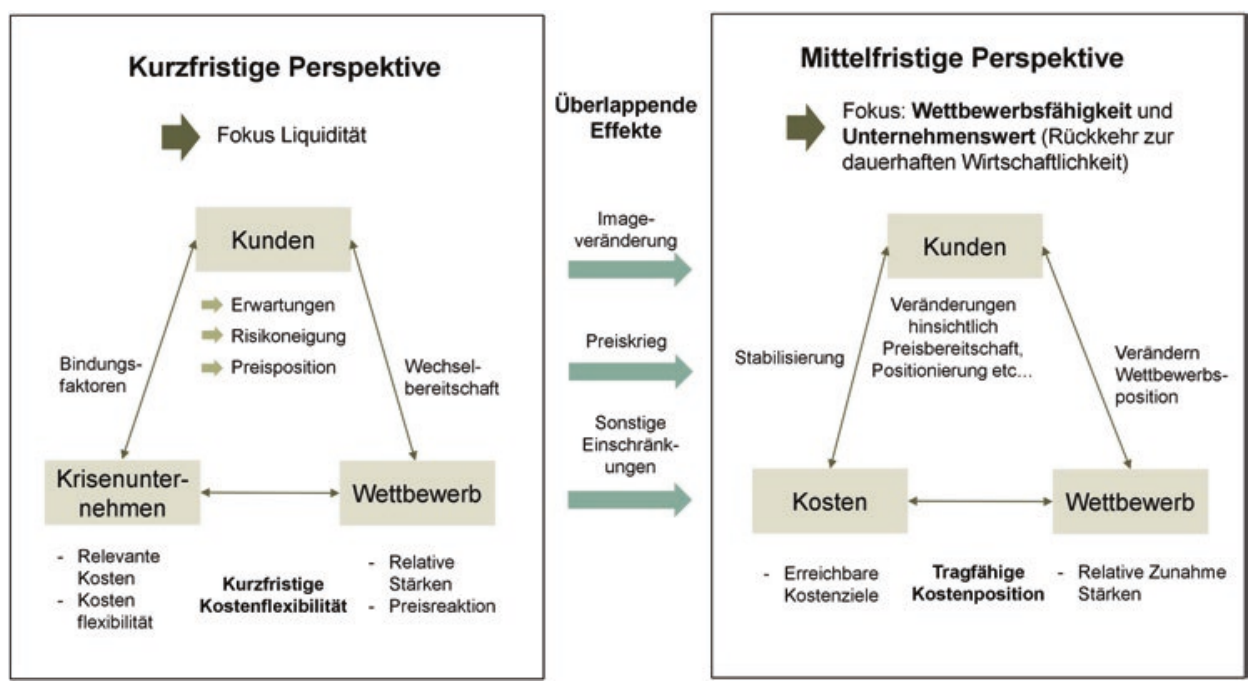

Abb. 4 Systematische Analyse preispolitischer Kommunikation - Spielräume in der Krise 
- Erwarten die Kunden möglicherweise Preisvariationen? Dies wird insbesondere dann der Fall sein, wenn die Kunden eine stärkere Position wahrnehmen und deshalb mit Preissenkungen rechnen.

- Ist aufgrund der Risikoneigung der Kunden damit zu rechnen, dass sie als Kompensation eines höheren Risikos bei der Zusammenarbeit mit einem Krisenunternehmen niedrigere Preise erwarten?

Die Antworten auf diese Fragen bilden nicht nur die Grundlage zur Erarbeitung einer Preisstrategie in der Krise, sondern geben auch einen Eindruck davon, inwiefern aktiv oder passiv kommuniziert werden muss. Stellt sich heraus, dass die Antworten auf diese Fragen allesamt auf eine hohe Bedeutung des Preises und die Notwendigkeit konkreter Aktionen in diesem Bereich während der Krise hindeuten, wird an einer aktiven Kommunikation, die diese Erwartungen aufnimmt, kein Weg vorbeiführen.

Wesentliche Nebenbedingung bei der Erarbeitung eines bestimmten kommunikativen Vorgehens sind die Bindungsfaktoren zum Krisenunternehmen bzw. die Wechselbereitschaft. Handelt es sich nur um einen Anbieter unter vielen (was schon Teil der Krisenursachen sein kann), so wird es schwierig werden, mit den Kunden gemeinsam über Sanierungsbeiträge oder Konditionenveränderungen zu sprechen. Situationen wie sie zum Beispiel manche Automobilzulieferer im Zuge der „Nachwehen“ zur Sub-Prime-Krise 2008/2009 erlebten, waren hingegen wesentlich günstiger für diese. Aufgrund geringerer Absatzzahlen und sich verschärfender Schwierigkeiten in der Unternehmensfinanzierung befanden sich zahlreiche Zulieferer in einer existenzbedrohenden Situation. Dadurch, dass innerhalb laufender Serien die Komponenten- und Teilelieferanten aber nicht ohne weiteres gewechselt werden konnten, bestand eine gewisse Bindung der Automobilhersteller. Von den Zahlungskonditionen bis hin zu Preisen konnten hier erhebliche Zugeständnisse vereinbart werden.

Wie flexibel Krisenunternehmen preislich sein können, hängt von der eigenen Kostensituation und deren Transparenz für das Management ab. Eine genaue Kenntnis der relevanten Kosten (also derjenigen, die auch ausgabenwirksam sind und die Liquiditätssituation beeinflussen) und der Flexibilität der eigenen Kosten (Kostenverlauf bei Mengenschwankungen und Möglichkeit des Kostenabbaus) sind Grundvoraussetzungen, um einschätzen zu können, inwiefern sich durch preispolitische Aktionen Verbesserungen oder Verschlechterungen der aktuellen Liquiditätssituation erzielen lassen. Hier ist für eine angemessene Einschätzung der Situation allerdings auch erforderlich, die Reaktionsmöglichkeiten des Wettbewerbs abzuschätzen. Der Ausnutzung der eigenen Reaktionschancen sind dort Grenzen gesetzt, wo Wettbewerber möglicherweise noch wesentlich flexibler reagieren können.

Insbesondere dann, wenn das Ziel der Sanierung in einem weiteren Betrieb des Unternehmens und eventuell dessen Verkaufs besteht, sind über die kurzfristige Perspektive hinaus auch mittelfristige Effekte zu analysieren. Dies ist dann nicht notwendig, wenn eine Liquidation und damit die Erzielung eines möglichst hohen Liquidationsergebnisses das Ziel ist. 
Überlappungen zwischen der kurz- und der mittelfristigen Betrachtung ergeben sich einige. Preisaktives Handeln in der Krise und eine entsprechende Kommunikation beeinflussen mittelfristig die Möglichkeiten des Krisenunternehmens. Veränderungen wirken nach, z. B. bei Image und Preispositionierung. Das Aufkommen von Preiskriegen, die durch intensive Preissenkungen und entsprechende Reaktionen des Wettbewerbs ausgelöst werden, kann zu dauerhaften Störungen im Markt führen. Eine Re-Dimensionierung des Unternehmens in der Krise verengt möglicherweise zukünftig das Angebot des Unternehmens, schränkt Vertrieb und Kommunikation über die Krise hinaus ein.

Eine Analyse der Stärke dieser Effekte und ihre Auswirkungen auf die im Fokus stehenden Ziele Wettbewerbsfähigkeit, nachhaltige Wirtschaftlichkeit und damit auch Unternehmenswert ist dann, wenn diese mittelfristige Perspektive von Bedeutung ist, der Gegencheck für kurzfristige Maßnahmen. Es gilt dann abzuwägen, was zum Überleben unbedingt notwendig ist bzw. was auch mittelfristig nachwirken könnte.

Bei dieser Betrachtung spielt die Kommunikation eine besondere Rolle. Je aktiver in der Krise kommuniziert wurde, desto höher ist die Gefahr, dass sich Botschaften der Krisenkommunikation im Markt verfestigt haben. So wesentlich und notwendig in vielen Fällen eine aktive Krisenkommunikation (wie oben dargestellt) auch ist, ihr Einsatz sollte auf den Fall des akuten „Überlebenskampfes“ beschränkt bleiben.

\section{Besonderheiten der Preiskommunikation in Branchen- oder gesamtwirtschaftlichen Krisen}

Ein Sonderfall der Krise (z.B. durch die COVID-19-Pandemie) sind Situationen, in denen kurzfristig zumindest komplette Branchen (z.B. Einzelhandel, Tourismus etc.) wenn nicht sogar das gesamte volkswirtschaftliche Umfeld in eine Rezession geraten. Alle relevanten Akteure befinden sich im Krisenmodus und nicht nur einzelne Unternehmen.

Die Gefahr besteht hier insbesondere in einem kommunikativen „Herdenverhalten“ mit z.T. dauerhaften Nachwirkungen. In der Branchenkrise der Luftfahrt, die schon vor COVID-19 begann und zu teilweise ruinösen Preiskriegen führte, kam es z.B. zu einer anhaltenden preislichen Umpositionierung des Fliegens bei potentiellen Fluggästen. „Signaling " ist deshalb gerade in solchen umfassenden Krisen sehr wesentlich: Eine begründete und offensiv kommunizierte eigene Zurückhaltung bei Preissenkungen kann ein wesentliches Signal auch in die eigene Branche hinein sein, auf preisliche Amokläufe zu verzichten. Begründungen können etwa die Sicherung der Qualität oder die (System-)Relevanz der eigenen Branche sein und die daraus resultierende Notwendigkeit wirtschaftlicher Stabilität durch auskömmliche Preise (als eine Art Beitrag aller zur Aufrechterhaltung bestimmter Angebote).

Das Vorgehen in der Konzeptionierung der Preiskommunikation unterscheidet sich generell nicht von dem zuvor geschilderten. Wesentlich sind die schonungslose Analyse und Zielsetzung. Hat das Unternehmen eine realistische Perspektive, die über die Krise hinausreicht, ist mit der Preiskommunikation wesentlich vorsichtiger umzugehen, als wenn die 
Krise wahrscheinlich die Liquidation des Unternehmens bedeuten wird. Im Falle eines geplanten Fortbetriebs sind bei der Konzeptionierung des Vorgehens die relative Liquiditätsstärke gegenüber anderen Anbietern und die Flexibilität im Abbau von Kapazitäten sowie der ausgabenwirksamen Fixkosten kritische Größen: Je mehr Spielraum ein Unternehmen hier aufweist, desto vielfältiger sind auch die Handlungsoptionen und desto eher ist es möglich, auch ohne aggressive Preiskommunikation zu überleben - was im Sinne des langfristigen Werterhalts des Unternehmens auf jeden Fall zu bevorzugen ist.

\section{$8 \quad$ Fazit}

Krisen stellen eine besondere Herausforderung der Preiskommunikation dar: Durch sich schlagartige verändernde Ziele und Rahmenbedingungen der Unternehmensführung - höhere Bedeutung der (kurzfristigen) Liquidität, möglicherweise Suche nach Investoren etc. einerseits und sich verändernde Stärkeverhältnisse im Dreieck Unternehmen, Kunden, Wettbewerb andererseits. War der Preis nicht ohnehin schon Teil der Krisenursachen, so kann er jetzt doch schnell zum Problem werden: Das Preisniveau zu halten, wird schwierig dadurch, dass Kunden Preiszugeständnisse erwarten. Sie wollen die Kompensation eines als höher eingeschätzten Risikos und einen Preis, der ihrer wahrgenommenen zusätzlichen Stärke entspricht. Dieser Preisdruck verschärft sich, wenn Wettbewerber neue Chancen durch die Abwerbung von Kunden des Krisenunternehmens erkennten und diese preisaggressiv umwerben. Dem Krisenunternehmen selbst liegen Preissenkungen aber häufig auch gar nicht fern: Es besteht die Verlockung, durch aggressive Preispolitik massiv den Absatz zu steigern. Sie wird allerdings durch eine wenig flexible Nachfrage und die mittelfristig starke, negative Nachwirkung einer solchen Vorgehensweise relativiert.

In diesem Beitrag wurde dargelegt, dass es einer konsistenten Preispolitik in der Krise bedarf, die auf die situativen Besonderheiten eingeht. Zu dieser Krisenstrategie ist das passende kommunikative Vorgehen zu wählen. Zur Entwicklung von Strategie und Kommunikation bedarf es eines stringenten Aktionsplans, der auf einer kurz- und mittelfristigen Analyse der preispolitischen Effekte an sich und im Zusammenhang beruht.

\section{Literatur}

Behringer S (2017) Unternehmenssanierung: Ursachen - Krisenfrüherkennung - Management. Springer, Wiesbaden

Diller H (2003) Preispolitik. Kohlhammer, Stuttgart

Evertz D, Krystek U (2014) Unternehmen erfolgreich restrukturieren und sanieren: Herausforderungen und Lösungsansätze für den Turnaround. Schäffer-Poeschel, Stuttgart

Fürst R (2004) Preiswettbewerb in Krisen: Auswirkungen der Terror-Attentate des 11. September 2001 auf die Luftfahrtbranche. Dt. Univ.-Verlag, Wiesbaden

Häger M, Hiltner E (2017) Gutachterliche Kommentierung IDW-S6, BGH-Rechtsprechung und Restrukturierungsplanung. In: Knecht T, Hommel U (Hrsg) Handbuch Unternehmensrestrukturierung: Grundlagen - Konzepte - Maßnahmen. Springer Gabler, Wiesbaden 
Helbing Gruppe (2009) Management Letter - Wege aus der Unternehmenskrise. Zürich, Helbing Corporate Finance AG. https://www.helbling.ch/news-archive-static/wege-aus-der-unternehmenskrise-HOL_Management-Letter_Wege-aus-der-Unternehmenskrise_012009.pdf. Zugegriffen am 03.05.2019

Homburg C (2017) Grundlagen des Marketingmanagements: Einführung in Strategie, Instrumente, Umsetzung und Unternehmensführung. Springer, Wiesbaden

IDW (2018) IDW-Standard: Anforderungen an Sanierungskonzepte. Institut der Wirtschaftsprüfer in Deutschland e.V. IDW, Düsseldorf

Katzdobler S (2014) Strategie und taktische Maßnahmen in Krisenzeiten: Eine Analyse über das Verhalten von Führungskräften in der Wirtschaftskrise. Springer, Wiesbaden

Klein A (2009) Kostenmanagement in Krisenzeiten: Instrumente zur Kostensenkung und Liquiditätsverbesserung. Haufe-Lexware, Freiburg

Longstaff F (2008) Train wrecks: asset pricing and the valuation of severely distressed assets. UCLA Anderson School and NBER, Los Angeles. http://w4.stern.nyu.edu/finance/docs/pdfs/Seminars/081f-longstaff.pdf. Zugegriffen am 20.04.2019

Meffert H (2013) Marketing Arbeitsbuch: Aufgaben - Fallstudien - Lösungen. Gabler, Wiesbaden

Plinke W (1991) Die Geschäftsbeziehung als Investition. In: Specht G, Silberer G, Engelhart W (Hrsg) Marketing-Schnittstellen. Schäffer-Poeschel, Stuttgart

Rao A, Bergen M, Davis S (2000) How to fight a Price War. https://hbr.org/2000/03/how-to-fight-aprice-war. Zugegriffen am 15.03.2019

Schüler A (2012) Bewertung sanierungsbedürftiger Unternehmen: Wertbausteine, Risikoverteilung und Kapitalkosten. Springer, Wiesbaden

Simon H, Fassnacht M (2009) Preismanagement: Strategie - Analyse - Entscheidung - Umsetzung. Springer Gabler, Wiesbaden

Spremann K (2005) Bewertung von Unternehmen im Financial Distress. Universität St. Gallen, St. Gallen. https://www.alexandria.unisg.ch/32443/1/FinancialDistress-1206.pdf. Zugegriffen am 05.03.2019

Thießen A (2013) Handbuch Krisenmanagement. Springer, Wiesbaden

Dr. Michael Paul (Jahrgang 1968) ist Gründer und Geschäftsführer von paul und collegen consulting in Wien und Berlin. Er widmet sich in der Beratung schwerpunktmäßig der Sanierung und Restrukturierung von Unternehmen sowie Distressed M\&A. Beginnend mit seiner Promotion hat er sich immer wieder mit Themen der Optimierung von Pricing und Vertrieb beschäftigt. Dass eine Sanierung auch diese Aktionsfelder im Auge behalten muss und nicht rein kostenorientiert erfolgen kann, ist eine wesentliche Erfahrung, die er in zahlreichen Projekten machen konnte.

Nach den Standards der EACVA ist er „Certified Valuation Analyst“ (CVA) für Unternehmensund Markenbewertungen und seit April 2016 allgemein beeideter und gerichtlich zertifizierter Sachverständiger für das Fachgebiet „Unternehmensbewertung, Unternehmensplanung“. Er ist Verfasser zahlreicher Beiträge in Fachzeitschriften und Autor fachspezifischer Bücher. 